\title{
Meta
}

Journal des traducteurs

Translators' Journal

GAMBIER, Y. and H. GoTTLIEB (eds.) (2001) : (Multi)Media

Translation, Concepts, Practices and Research, Amsterdam/Philadelphia, John Benjamins, Benjamins

Translation Lilbrary, vol. 34, XX-298 p.

\section{Christian Balliu}

Volume 48, numéro 3, septembre 2003

Traduction et enseignement

Translation and teaching

URI : https://id.erudit.org/iderudit/007606ar

DOI : https://doi.org/10.7202/007606ar

Aller au sommaire du numéro

Éditeur(s)

Les Presses de l'Université de Montréal

ISSN

0026-0452 (imprimé)

1492-1421 (numérique)

Découvrir la revue

Citer ce compte rendu

Balliu, C. (2003). Compte rendu de [GAMBieR, Y. and H. GotTLieb (eds.) (2001) : (Multi)Media Translation, Concepts, Practices and Research,

Amsterdam/Philadelphia, John Benjamins, Benjamins Translation Library, vol.

34, XX-298 p.] Meta, 48(3), 450-453. https://doi.org/10.7202/007606ar 


\section{Comptes rendus}

Gambier, Y. and H. Gottlieb (eds.) (2001): (Multi)Media Translation, Concepts, Practices and Research, Amsterdam/Philadelphia, John Benjamins, Benjamins Translation Library, vol. 34, xx-298 p.

Cet ouvrage reprend 26 articles, extraits de l'ensemble des exposés présentés lors de deux rencontres, le Séminaire de Misano en 1997 et le Colloque de Berlin en 1998. Les conférenciers sont issus de nombreux pays, dont les États-Unis, l'Espagne, la Belgique, le Portugal, la Pologne, la Grèce et l'Angleterre. Le Séminaire de Misano avait pour thème Multimedia and Translation et le Colloque de Berlin était consacré à Quality and Standards in Audiovisual Language Transfer.

Le titre du livre est très révélateur : les éditeurs ont choisi de l'intituler (Multi)Media Translation, indiquant d'entrée le passage presque naturel des médias au multimédia et la volonté d'étudier les deux activités ensemble, comme un tout homogène, au sein de la traductologie. S'il est vrai que le sujet est à la mode, il est tout aussi vrai que le nombre d'études qui y sont consacrées restent peu nombreuses. C'est à cette lacune que les organisateurs du colloque et les éditeurs ont voulu remédier en rassemblant des praticiens d'horizons très différents (traducteurs et interprètes professionnels, enseignants, spécialistes du sous-titrage, des médias et de la communication, etc.) et en colligeant des points de vue très variés.

Comme les éditeurs se plaisent à le souligner dans leur «Avant-propos», les nouvelles technologies de l'information et de la communication changent non seulement notre vie quotidienne, mais modifient aussi notre perception de la langue, tout en favorisant la diffusion des connaissances et le développement des identités culturelles. Ce fait est pourtant encore peu pris en considération et on peut même dire que la localisation semble échapper pour le moment aux professionnels de la traduction ainsi qu'à l'enseignement universitaire qui devrait s'y intéresser. Outre son apport théorique non négligeable, ce livre propose ainsi une réflexion sur un possible recentrage de la traduction et lance dans tous les cas un cri d'alarme.

Une des raisons de ce phénomène est à rechercher dans la crise d'identité que traverse actuellement l'activité traduisante et qui se manifeste d'ailleurs dans les différents secteurs qui s'occupent aujourd'hui de traduction au sens large. Ces secteurs préfèrent qualifier leurs activités autrement: localisation, transfert linguistique, adaptation, révision, médiation langagière, etc. Sans doute parce qu'ils ne font pas appel à des traducteurs au sens classique du terme, ces derniers étant assimilés à un simple transcodage linguistique ou semblant démunis face à des «textes» qui ne sont plus une somme d'éléments linguistiques, mais qui intègrent des images, du son ou des graphiques.

On peut dire que depuis le $\mathrm{xv}^{\mathrm{e}}$ siècle, le texte imprimé est le lot quotidien du traducteur. Il l'a été pendant près de six siècles, et ce n'est qu'à la fin du $\mathrm{xx}^{\mathrm{e}}$ siècle que

Meta, XLVIII, 3, 2003 
le support a radicalement changé, grâce aux progrès de l'informatique. Ne pas adapter les filières de formation en ce sens, ne pas proposer des cours de formation continue signifierait à terme écarter les traducteurs de l'univers de plus en plus polymorphe de la traduction. Une véritable aliénation par manque d'adaptation.

Tout cela demande, les éditeurs ont raison, une étude de marché pour prédire les besoins de demain et, en conséquence, les compétences des futurs traducteurs que les écoles formeront. Cela demande aussi un effort théorique et terminologique pour préciser les champs d'application de cet éventail d'activités. On peut raisonnablement affirmer que les traductions audiovisuelle et multimédia partagent quatre caractéristiques:

1. le travail d'équipe;

2. la présence de «textes» intermédiaires (scénarios, scripts, etc.) qui rendent caduc le distinguo classique entre texte source et texte cible;

3. l'importance des critères de lisibilité, d'accessibilité et d'utilité;

4. la remise en question de l'enseignement traditionnel de la traduction, en raison du fossé de plus en plus béant entre universités et monde professionnel.

L'interdisciplinarité est sans doute le mot-clé de l'ouvrage, tous les auteurs insistant sur l'éventail des compétences à mettre en œuvre dans la traduction (multi)média. Ce livre est aussi un appel à une ouverture de la traductologie à des domaines annexes, comme la sémiologie, la sociologie, l'anthropologie, les sciences de l'information, l'informatique ou encore les métiers du cinéma. Edmond Cary ne disait-il pas déjà cela à l'aube des années 1960, lorsqu'il évoquait le polymorphisme de la profession?

Il est évident que la transformation du marché de l'audiovisuel pose de nouvelles exigences et demande par conséquent de nouvelles compétences traduisantes. Il suffit de penser aux besoins accrus de traduction de pages Web, à la localisation, sans parler des offres de traduction sur Internet. La traduction automatique proposée gratuitement en ligne ne fait que multiplier les contenus, grâce à des systèmes comme Systran ou Easy Translator. Il est vrai que la qualité générale offerte par les systèmes de traduction automatique prête à sourire, mais les versions produites sont de plus en plus utilisables et permettent un gain appréciable en rapidité et en disponibilité.

Pour ce qui est de la localisation, on trouve pour le moment deux formules: soit la collaboration entre traducteurs, informaticiens, spécialistes du domaine, soit, mais c'est plus rare, l'ensemble du travail est confié au localisateur, ce traducteur en prise avec son époque, qui a su s'adapter à toutes les facettes, traduisantes, techniques, juridiques, de ce nouveau métier. Avec le multimédia, tous les sujets sont possibles: la cuisine, la médecine, l'impressionnisme, les contes de Perrault, la France de Louis XIV, le cinéma, le génie génétique, les icônes, etc.

Un autre élément qui mérite réflexion est que la localisation ne concerne pas les seuls textes écrits, invalidant par la même occasion la distinction habituelle entre interprétation et traduction ou entre traduction orale et traduction écrite. Qu'il s'agisse de cinéma, de CD-ROM ou de Web, les textes oraux sont tout aussi présents que les textes écrits. Il s'agit alors non seulement de traduire, mais aussi de rendre des voix, des musiques, des sonorités; en un mot, d'adapter. Il y a aussi les images, notamment dans les messages publicitaires, qu'il convient d'harmoniser avec la culture d'arrivée. Par exemple, il m’a été raconté que dans une publicité destinée au monde arabe, la 
marque de montres Tissot a dû retirer le drapeau suisse qui orne généralement ses annonces pour ne pas heurter la communauté d'accueil où ce drapeau pouvait être assimilé à la croix chrétienne, avec un éventuel impact négatif sur le chiffre de ventes et sur l'image de la marque. Finalement, outre l'importance de plus en plus prégnante des connaissances techniques et informatiques, la culture reste le viatique indispensable de tout enseignement de traduction digne de ce nom. On pourrait presque dire que l'on a toujours localisé, si c'est d'adaptation à la culture cible dont il s'agit. La seule chose qui ait vraiment changé, c'est le moyen.

Un changement de cap est cependant en train de s'opérer dans le domaine des langues de travail. Si l'anglais reste, et de loin, la langue dominante, notamment sur le Web, la localisation permet aux autres langues, y compris des langues plus confidentielles, de s'affirmer également et de lutter ainsi contre l'uniformisation anglo-saxonne, sur le plan linguistique du moins... Un autre effet linguistique est induit de ces nouvelles techniques, et il se remarque le plus en matière de soustitrage: l'entretien de la compétence linguistique et l'apprentissage de nouvelles langues étrangères. Un enquête de 1997 a montré que $75 \%$ des téléspectateurs de BBC World regardent cette chaîne pour améliorer leur niveau d'anglais. Les responsables de F3, de TV5 et de TV4 (chaîne suédoise) ont compris l'importance des sous-titres intralinguaux, c'est-à-dire dans la langue du pays. Comme le remarquent avec raison les éditeurs, les sous-titres intralinguaux et interlinguaux consolident les compétences en lecture; en fait, nous lisons la télévision. À titre personnel, je considère qu'Internet, avec ses multiples possibilités, comme le courrier électronique, détourne beaucoup moins les gens de la lecture et de l'écriture qu'on ne le pense. Il y a un effet rebond, dirait-on en médecine, qui fait que l'on écrit et que l'on lit peutêtre davantage qu'avant, mais sans s'en rendre compte! En réalité, c'est surtout le support qui change; on est passé du papier, support privilégié pendant si longtemps, à l'écran, qui est devenu le nouveau support de prédilection depuis la seconde moitié du $\mathrm{xx}^{\mathrm{e}}$ siècle.

L'image du traducteur ne se résume plus à celui d'intermédiaire entre un texte de départ et un texte d'arrivée. S'il est toujours, et plus que jamais, un passeur de cultures, son activité quotidienne dépasse les conventions textuelles pour prendre en compte toute la sémiologie des interactions socioculturelles, en passant par d'autres codes et d'autres canaux de communication. Le rapport du texte à l'image et au son, le mélange de l'oral et de l'écrit, la variété des supports et le recours de plus en plus grand à l'hypertextualité sont en train de modifier en profondeur les compétences et l'horizon de travail du traducteur. Une fois encore, c'est le marché, qui fut pendant longtemps un mot exclu des études traductologiques, qui dicte sa loi.

Si la demande change, la réflexion théorique - qui est l'objectivation des pratiques - doit elle aussi se mettre au diapason et sortir de sa gangue linguistique pour adopter une approche polysystémique et intégrer des éléments de psychologie cognitive. C'est sans doute le défi le plus difficile à relever.

Comme l'ouvrage le montre à suffisance, la traduction devient aujourd'hui, et de manière inéluctable, non verbale, ce qui rend justice à distance à ces précurseurs qui avaient pressenti que traduire n'est pas une affaire de mots. De là à ce que la traduction pour les médias ou la traduction multimédia deviennent des disciplines à part entière, distinctes de la traduction au sens historique, il y a plus qu'un pas. 
Malgré quelques redites inévitables dans ce genre d'exercice, le livre (Multi)Media Translation, qui contient de nombreuses références et une bibliographie abondante, séduira ceux qui s'intéressent à cette nouvelle manière de traduire et souhaitent actualiser leurs connaissances.

Christian Balliu

Haute École de Bruxelles, Bruxelles, Belgique

Bowker, L. (2002) : Computer-Aided Translation Technology: A Practical Introduction, Ottawa, University of Ottawa Press, Didactic of Translation Series, xx-185 p.

Lynne Bowker, professeure à l'École de traduction et d'interprétation de l'Université d'Ottawa, propose ici un manuel d'introduction à la traduction assistée par ordinateur (TAO - angl. Computer-Aided Translation, CAT). Il s'agit d'un ouvrage compact et très ciblé dans son contenu: il se concentre sur la présentation des outils de TAO du point de vue de leur potentiel d'utilisation par les traducteurs. Il s'adresse donc avant tout aux étudiants et professeurs en traduction ainsi qu'aux professionnels du domaine désireux de mettre à jour leurs connaissances. De par son contenu et le public visé, ce manuel se rapproche de l'ouvrage de L'Homme (1999), un ouvrage plus global (en français), qui couvre l'ensemble des outils informatiques utilisés en traduction et non la seule TAO. Puisque le livre de Lynne Bowker est un manuel (une succession de leçons), il sera présenté ici de façon linéaire, ce compte rendu se terminant par une évaluation générale.

L'«Introduction» présente le contenu du manuel, le public visé et l'intérêt potentiel de l'étude de la TAO. Notamment, l'auteure justifie le fait de se focaliser sur la TAO, en situant celle-ci dans le contexte économique actuel. La distinction entre traduction automatique (TA - angl. Machine Translation, MT) et TAO est clairement présentée et, surtout, l'intérêt économique et professionnel (pour le traducteur) de la TAO est bien justifié vis-à-vis de la TA. L'auteure démontre notamment pourquoi le relatif échec de l'utilisation commerciale de la TA, en regard des espoirs et des investissements qu'elle a suscités, ne doit pas influencer la façon dont on peut envisager la TAO. Cette dernière, outre le fait qu'elle est maintenant omniprésente dans la pratique quotidienne du traducteur, est devenue incontournable pour qui veut rester compétitif dans sur le marché de la traduction professionnelle. Dans l'«Introduction», l'auteure justifie aussi le choix des technologies qui seront présentées. Il s'agit exclusivement des outils informatiques ayant une importance particulière pour la profession de traducteur; cela exclut donc les outils d'usage courant mais non spécifiquement liés à cette activité professionnelle (traitement de texte, navigation sur Internet, etc.).

Si l'«Introduction» est stimulante, le Chapitre 1 fait un peu retomber la sauce. Il est consacré à une présentation des raisons pour lesquelles il est utile aux traducteurs d'acquérir la connaissance des outils technologiques. Outre le fait que certains arguments en ce sens sont mentionnés dans l' "Introduction», on pourrait penser que ce chapitre, de par son contenu, a tout à fait sa place comme section de l'«Introduction», auquel il me semble logiquement appartenir.

Le Chapitre 2 introduit les technologies de conversion des données papier en données électroniques. En d'autres termes, l'auteure y présente le fonctionnement et 